DRAFT VERSION MARCH 28, 2018

Preprint typeset using $\mathrm{LAT}_{\mathrm{E}} \mathrm{X}$ style AASTeX6 v. 1.0

\title{
CENTRAL ENGINE-POWERED BRIGHT X-RAY FLARES IN SHORT GAMMA-RAY BURSTS: A HINT OF BLACK HOLE-NEUTRON STAR MERGER?
}

\author{
Hui-Jun Mu${ }^{1}$, Wei-Min Gu${ }^{1}$, Jirong MaO ${ }^{2,3,4}$, Shu-Jin Hou ${ }^{5}$, DA-Bin Lin ${ }^{6}$, And Tong Liu ${ }^{1}$
}

(Received; Revised; Accepted)

\footnotetext{
${ }^{1}$ Department of Astronomy, Xiamen University, Xiamen, Fujian 361005, China

${ }^{2}$ Yunnan Observatories, Chinese Academy of Sciences, 650011 Kunming, Yunnan Province, China

${ }^{3}$ Center for Astronomical Mega-Science, Chinese Academy of Sciences, 20A Datun Road, Chaoyang District, Beijing, 100012, China

${ }^{4}$ Key Laboratory for the Structure and Evolution of Celestial Objects, Chinese Academy of Sciences, 650011 Kunming, China

${ }^{5}$ College of Physics and Electronic Engineering, Nanyang Normal University, Nanyang, Henan 473061, China

${ }^{6}$ Guangxi Key Laboratory for Relativistic Astrophysics, Department of Physics, Guangxi University, Nanning 530004, China
}

\begin{abstract}
Short gamma-ray bursts may originate from the merger of double neutron stars (NS) or that of a black hole $(\mathrm{BH})$ and an NS. We propose that the bright X-ray flare related to the central engine reactivity may hint a BH-NS merger, since such a merger can provide more fall-back materials and therefore a more massive accretion disk than the NS-NS merger. Based on the observed 49 short bursts with Swift/X-ray Telescope follow-up observations, we find that three bursts have bright X-ray flares, among which three flares from two bursts are probably related to the central engine reactivity. We argue that these two bursts may originate from the BH-NS merger rather than the NS-NS merger. Our suggested link between the central engine-powered bright X-ray flare and the BH-NS merger event can be checked by the future gravitational wave detections from advanced LIGO and Virgo.
\end{abstract}

Keywords: accretion, accretion disks — gamma-ray burst: general — X-rays: general

\section{INTRODUCTION}

Gamma-ray bursts (GRBs) are generally divided into two classes in the $T_{90}$ (duration) - HR (spectral hardness) space: long-soft GRBs (LGRBs) and short-hard GRBs (SGRBs) (Kouveliotou et al. 1993), or physically distinct types: Type I and II GRBs (Zhang et al. 2009). It is well-known that LGRBs are associated with type Ib/c supernovae (SNe), which are generally triggered by the collapse of massive stars (for a review, see Woosley \& Bloom 2006). SGRBs originate from the merger of double neutron stars (NS) or that of a black hole (BH) and an NS (e.g., Paczynski 1986, 1991; Eichler et al. 1989; Narayan et al. 1992).

The compact binary mergers (NS-NS, BH-NS, BH-BH) are the promising sources of strong gravitational waves (GWs). Before the discovery of the GW event from the NS-NS merger, the binary NS system had been detected in our Galaxy through electromagnetic (EM) radiation (e.g., Tauris et al. 2017). After the first detection of high-frequency GW signals from the BH-BH merger (e.g., Abbott et al. 2016a,b), a new era of astronomy has been launched. However, since the merger of BH-BH is not expected to produce EM signals, a wide range of EM counterparts are expected for the NS-NS and BH-NS mergers (Metzger \& Berger 2012; Zhang 2016; Liu et al. 2017). On August 17, 2017, the Laser Interferometer Gravitational-Wave Observatory (LIGO) and the Virgo detector simultaneously detected a transient GW event of an NS-NS merger (GW 170817; Abbott et al. 2017). SGRB 170817 was independently detected with a delay of about 1.7s by both Fermi Gamma Ray Burst Monitor (Goldstein et al. 2017; Zhang et al. 2018) and INTEGRAL (Savchenko et al. 2017). This is the first specific GW signals from the NS-NS merger, and with an EM counterpart for the first time.

The ejection of neutron-rich matter is another key prediction of the compact binary merger. The r-process-enriched ejecta (the so-called "mini-SN", "kilonovae" or "macronova"; see e.g., Li \& Paczyński 1998; Metzger et al. 2010; Barnes \& Kasen 2013; Yu et al. 2013; Metzger 2017; Kasen et al. 2017) ${ }^{1}$ from NS-NS or BH-NS mergers was widely

\footnotetext{
guwm@xmu.edu.cn

1 Recently, by testing the BH hyperaccretion (for a review, see Liu et al. 2017) inflow-outflow model for powering GRBs, Liu et al. (2018) and Song \& Liu (2017) proposed that another type of "nova" after the merger event may be detected. In addition, Ma et al. (2018) proposed a Blandford-Payne mechanism-powered merger-nova in the $\mathrm{BH}$ hyperaccreting.
} 
studied (e.g., Jin et al. 2016). On the other hand, the simulations of NS-NS mergers (e.g., Dietrich et al. 2015) and BH-NS mergers (e.g., Siegel \& Metzger 2017) showed an upper limit of the remnant disk mass, i.e, $\sim 0.3 M_{\odot}$. The BH-NS merger simulations proposed that the typical ejecta velocity is similar to the NS-NS case. However, the ejecta mass $M_{\mathrm{ej}}$ generally increases with larger asymmetry in the mass ratio, which can reach $0.1 M_{\odot}$ in the $\mathrm{BH}-$ NS merger (Kawaguchi et al. 2015, 2016). According to the BH-NS merger model presented in Tanaka et al. (2014) and Kawaguchi et al. (2016), the ejecta mass can be estimated, i.e., GRB 050729 (I-band data, $M_{\mathrm{ej}} \sim 0.05 M_{\odot}$ ), GRB 060614 (I-band data, $M_{\mathrm{ej}} \sim 0.1 M_{\odot}$ ), and GRB 130603B (H-band data, $M_{\mathrm{ej}} \sim 0.03 M_{\odot}$ ). The massive ejecta $\left(\gtrsim 0.05 M_{\odot}\right)$ suggests that the progenitor of GRB 050709 is the BH-NS merger (Jin et al. 2016), which is similar to the macronova candidate of GRB 060614 (Yang et al. 2015; Jin et al. 2015).

In this work, we propose that the bright X-ray flares after the prompt gamma-ray emission can also be a good probe for distinguishing BH-NS and NS-NS mergers. Several ideas have been proposed to explain the episodic phenomenon of X-ray flares, i.e., related to the internal dissipation (e.g., Perna et al. 2006; Dai et al. 2006; Rosswog 2007; Liu et al. 2017), or to the external shock (e.g., Proga \& Zhang 2006; Giannios 2006). The BH-NS merger simulations proposed that the typical ejecta mass can reach $0.1 M_{\odot}$ (Kawaguchi et al. 2015, 2016). Rosswog (2007) shows a larger spread in the fallback behavior in the BH-NS system than in the NS-NS one. For an SGRB having bright X-ray flare with internal origin, a more massive disk is required, corresponding to the BH-NS merger. Our suggested link between the central engine-powered bright X-ray flare and the BH-NS merger can be checked by the GW detectors. The remainder is organized as follows. Our data analysis and physical origin of the X-ray flares are presented in Section 2. The accreted mass for X-ray flares is investigated in Section 3. A comparison of NS-NS and BH-NS mergers is studied in Section 4. Conclusions and discussion are presented in Section 5.

\section{DATA ANALYSIS AND PHYSICAL ORIGIN}

In a recent review, Berger (2014) provides 67 Swift SGRBs, among which 31 events have X-Ray Telescope (XRT) rapid follow-up observations $(\lesssim 100 \mathrm{~s})$ and the adequate observational data in the early time $(100 \mathrm{~s} \text { to } 1000 \mathrm{~s})^{2}$. In addition, we include 18 other Swift SGRBs between January 2013 to August $2017^{3}$ with rapid and adequate XRT follow-up observations in our sample. We have examined the total 49 SGRBs in our sample, and searched for the bright X-ray flares satisfying the condition " $F_{\mathrm{p}}>3 F^{\prime}$ " (e.g., Mu et al. 2016), where $F_{\mathrm{p}}$ and $F$ are the peak flux and the underlying continuum flux at the peak time, respectively. Three SGRBs $(050724,131004 \mathrm{~A}$ and 161004A) are found to have bright X-ray flares, which are presented in Figure 1. In this figure, the single power-law model (black solid line) is adopted to fit the smooth continuum contribution (e.g., Bernardini et al. 2011; Margutti et al. 2011). All the X-ray light curves of the 49 SGRBs are presented in Figure 2, where $z=0.5$ (Berger 2014) is adopted for calculating the luminosity of the unknown redshift sources. The three SGRBs (050724, 131004A and 161004A) are shown by red, green and blue stars, respectively, and the other sources are shown by gray curves.

An empirical function proposed by Norris et al. (2005) is used for fitting the $0.3-10 \mathrm{keV}$ (Swift/XRT) X-ray flare light curve, i.e.,

$$
F_{t}=A e^{2\left(\tau_{1} / \tau_{2}\right)^{1 / 2}-\frac{\tau_{1}}{t}-\frac{t}{\tau_{2}}} .
$$

The peak time of the flare is $t_{\mathrm{p}}=\left(\tau_{1} \tau_{2}\right)^{1 / 2}$. Thus, the peak count rate of the flare is $A=F_{\max }=F_{t_{\mathrm{p}}}$. The width of the bright flare is measured between the two $1 / e$ intensity points,

$$
\omega=\Delta t_{1 / e}=\tau_{2}(1+4 \mu)^{1 / 2} .
$$

where $\mu=\left(\tau_{1} / \tau_{2}\right)^{1 / 2}$. The asymmetry of the flare is

$$
k=\frac{t_{\mathrm{d}}-t_{\mathrm{r}}}{t_{\mathrm{d}}+t_{\mathrm{r}}}=(1+4 \mu)^{-1 / 2} .
$$

where $t_{\mathrm{d}}=\omega(1+k) / 2$ and $t_{\mathrm{r}}=\omega(1-k) / 2$ are the decay and the rise time of the flare, respectively (Bernardini et al. 2011). The fitting results of the X-ray flares are shown in Table 1. The fitting procedure of the three SGRBs in our sample are shown in Figure 1. Results of the temporal evolution of the spectral photon index $\Gamma^{4}$ are portrayed in Figure 1.

In order to investigate the physical origin of X-ray flares, Ioka et al. (2005) proposed some limits on the timescale and amplitude of variabilities to identify the sources of afterglow variability, especially for $\omega<t_{\mathrm{p}}$. It is a useful tool to

\footnotetext{
${ }^{2}$ http://www.swift.ac.uk/xrtcurves/ (Evans et al. 2007, 2009) and http://www.astro.caltech.edu/grbox/grbox.php

3 http://www.astro.caltech.edu/grbox/grbox.php

4 http://www.swift.ac.uk/burst_analyser/
} 
trace the regions of allowance for the timescale and amplitude of variabilities bumps or flares in GRB afterglow on the basis of kinematic arguments. In Figure 3 we present our bright X-ray flares in the "Ioka plot" to judge the physical origin of the X-ray flares, which is based on the relative variability flux $\Delta F / F$ and the relative variability timescale $\omega / t_{\mathrm{p}}$. The X-ray flare candidates in SGRBs from Margutti et al. (2011) are also shown for the comparison. Since X-ray flares generally follow a fast rise exponential decay profile with $\omega \ll t_{\mathrm{p}}$, the criterion of $\omega<t_{\mathrm{p}} / 2$ is adopted for the central engine-powered X-ray flares. Similar to Ioka et al. (2005), the fraction of cooling energy $f_{c} \sim 1 / 2$ and $F /\left(\nu F_{\nu}\right) \sim 1$ are adopted for the X-ray band. As shown in Figure 3, the bright X-ray flares from GRBs 131004A and $161004 \mathrm{~A}$ are well located in the upper left region (above the green or red solid line and left to the cyan solid line), which indicates that the flares are likely to be related to the central engine reactivity. The fitting parameters are shown in Table 1.

On the other hand, by setting the zero time at the trigger time of GRB, $\alpha_{\text {dec }}=2+\beta$ is the maximum decay index for the external shock-powered flares (e.g., Kumar \& Panaitescu 2000; Liang et al. 2006; Kobayashi \& Zhang 2007), where $\beta$ is the spectral index. Then, $\alpha_{\mathrm{dec}}>2+\beta$ is taken as another criterion for the internal origin (Ioka et al. 2005; Bernardini et al. 2011; Mu et al. 2016). An even simpler criterion takes the form $\alpha_{\mathrm{dec}}>3$, since $\beta$ is usually around unity. The light curve index $\alpha_{\mathrm{dec}}$ in the decay phase (from $t_{\mathrm{p}}$ to $t_{\mathrm{p}}+t_{\mathrm{d}}$ ) can be estimated as

$$
\alpha_{\mathrm{dec}}=\frac{\log (e)}{\log \left[\left(t_{\mathrm{p}}+t_{\mathrm{d}}\right) / t_{\mathrm{p}}\right]},
$$

The spectral index $\beta$ in the decay phase of the flare is based on a power-law spectral model ${ }^{5}$. The spectral analyses results are reported in Table 1. In Figure 4, we present the flares in the $\alpha_{\mathrm{dec}}-\beta$ space. It is seen from Figure 4 that, the flares from GRBs 131004A and 161004A are located above the red solid line and the black dashed line, which implies that they are likely to be of the internal origin. Such a result is in good agreement with Figure 3. We therefore argue that the flares from GRBs 131004A and 161004A are related to the reactivity of the central engine.

\section{ACCRETED MASS FOR X-RAY FLARES}

For the central engine-powered bright flares in GRBs 131004A and 161004A, the isotropic energy of the flare $E_{\mathrm{X} \text {,iso }}$ in Swift/XRT energy range (0.3-10 keV) is calculated by

$$
E_{\mathrm{X}, \text { iso }}=\frac{4 \pi D_{\mathrm{L}}^{2} S_{\mathrm{F}}}{1+z}
$$

where $D_{\mathrm{L}}$ is the luminosity distance of GRB. The energy fluence in the energy range is

$$
S_{\mathrm{F}}=N \int_{0.1 t_{\mathrm{s}}}^{10 t_{\mathrm{e}}} A e^{2\left(\tau_{1} / \tau_{2}\right)^{1 / 2}-\frac{\tau_{1}}{t}-\frac{t}{\tau_{2}}} d t
$$

where $N$ is the factor of count-rate light curves converting into flux, $t_{\mathrm{s}}$ and $t_{e}$ are the two $1 / e$ intensity points, i.e., the start and the end time of the flare. We choose a sufficiently large time interval, i.e., from $0.1 t_{\mathrm{s}}$ to $10 t_{\mathrm{e}}$, for the integration of Equation (6). For GRB 161004A without redshift measurement, we adopt the median redshift of the SGRB population $z=0.5$ (Berger 2014). The isotropic peak luminosity of the flare is

$$
L_{\mathrm{p}}=4 \pi D_{\mathrm{L}}^{2} A N .
$$

The isotropic energy and peak luminosity of X-ray flares are reported in Table 2. The Blandford-Znajek (BZ) process (Blandford \& Znajek 1977) is a well-known mechanism to power GRBs and corresponding X-ray flares. Based on a common assumption that the magnetic field $B$ is around 10\% of its equipartition value (e.g., Popham et al. 1999; Di Matteo et al. 2002), the analytic BZ jet power ${ }^{6}$ can be expressed as (e.g., Popham et al. 1999; Lee et al. 2000a,b; Di Matteo et al. 2002; Lei et al. 2017)

$$
\dot{E}_{\mathrm{BZ}}=\lambda\left(a_{*}\right) \times 10^{51}\left(\frac{\dot{M}_{\mathrm{in}}}{M_{\odot} \mathrm{s}^{-1}}\right) \operatorname{erg~s}^{-1},
$$

where $\lambda\left(a_{*}\right)$ is a function of $a_{*}$. Following Lei et al. (2017) (but for a different ratio to the equipartition value), we obtain $\lambda\left(a_{*}\right) \simeq 1.8,3.1$ and 4.4 for $a_{*}=0.8,0.9$, and 0.95 , respectively. $\dot{M}_{\text {in }}=M_{\text {frag }}(1+z) / \omega$ is the average mass

\footnotetext{
5 http://www.swift.ac.uk/xrtspectra/addspec.php/

6 Since the neutrino annihilation mechanism may play an important role only for extremely high mass accretion rates, i.e., above the igniting accretion rate of an NDAF (e.g., Janiuk et al. 2007, 2013; Xue et al. 2013; Just et al. 2016; Lei et al. 2017), here we focus on the BZ (Blandford \& Znajek 1977) mechanism, which is valid for a wide range of mass accretion rates, even valid for sub-Eddington accretion systems such as X-ray binaries and AGNs.
} 
accretion rate for the X-ray flares, with $M_{\text {frag }}$ being the fragment mass. In addition, we assume that the BZ jet power $\dot{E}_{\mathrm{BZ}}$ roughly equals the isotropic luminosity $L_{\mathrm{X} \text {, iso }}$ of the flare (e.g., Liu et al. 2018), which means that the effects of the jet opening angle and the efficiency from the jet power to the jet luminosity are comparable. Then, the theoretical total energy of the X-ray flare can be expressed as

$$
E_{\mathrm{X}, \text { iso }}=L_{\mathrm{X}, \text { iso }} \frac{\omega}{1+z} \approx \dot{E}_{\mathrm{BZ}} \frac{\omega}{1+z}=\lambda\left(a_{*}\right) \times 10^{51} \frac{M_{\mathrm{frag}}}{M_{\odot}} \mathrm{erg} .
$$

A comparison of the theoretical results with the observations is shown in Figure 5, where the spin parameter is chosen as $a_{*}=0.8$ (red), 0.9 (black), 0.95 (green). It is seen from Figure 5 that, the BZ process can be responsible for the three flares in our sample. For a typical spin parameter $a_{*}=0.9$, the black dashed line shows that the required fragment mass is $\sim 0.01 M_{\odot}$ for each of the two flares of GRB $131004 \mathrm{~A}$, and $\sim 0.025 M_{\odot}$ for the flare of GRB $161004 \mathrm{~A}$.

\section{A COMPARISON OF NS-NS AND BH-NS MERGERS}

A hyper-accreting stellar-mass BH or a millisecond magnetar (e.g., Dai \& Lu 1998; Zhang \& Mészáros 2001) is usually invoked as the possible central engine of GRB. This work will mainly focus on the hyper-accreting BH. In the hyper-accreting stellar-mass $\mathrm{BH}$, a large amount of neutrinos can escape from the flow, namely neutrino-dominated accretion flow (for a review of NDAF, see Liu et al. 2017). Due to the high mass density of the NDAF, Liu et al. (2014) presented the effects of self-gravity on the vertical structure and the neutrino luminosity of the NDAF. The instabilities of a hyperaccreting disk around a $\mathrm{BH}$ may be responsible for the central engine-powered bright X-ray flare. The well-known disk instabilities, e.g., the thermal instability:

$$
\left(d \ln Q^{+} / d \ln T\right)_{\Sigma}>\left(d \ln Q^{-} / d \ln T\right)_{\Sigma}
$$

where $Q^{+}$and $Q^{-}$are the heating and cooling rates, respectively; the viscous instability:

$$
d \dot{m} / d \Sigma<0
$$

and the gravitational instability: the Toomre parameter

$$
Q_{\mathrm{T}}<1 .
$$

Di Matteo et al. (2002) suggested that the GRB disks are thermally stable and viscously stable (see their Figure 4 for the stability analysis). They also showed that $Q_{\mathrm{T}}$ decreases with increasing $r$, so that the flows are most unstable in the outer regions. However, only for the largest accretion rate $\dot{M} \sim 10 M_{\odot} \mathrm{s}^{-1}$ and for $R \gtrsim 50 R_{\mathrm{s}}$ does $Q_{\mathrm{T}}<1$, signifying gravitational instability (Popham et al. 1999; Narayan et al. 2001; Di Matteo et al. 2002; Perna et al. 2006). The gravitational instability may be the most likely candidate for the large-amplitude variability (Perna et al. 2006). Moreover, Perna et al. (2006) discussed the physical conditions in the outer parts of the hyperaccretion disk, and conclude that gravitational instability, possibly followed by the actual fragmentation of the disk, is the most likely candidate for the large-amplitude variability of the central engine output of both LGRBs and SGRBs.

The scenario is that, since the GRB disk is thermally and viscously stable (Popham et al. 1999; Narayan et al. 2001; Di Matteo et al. 2002), the gravitational instability may be the most likely candidate for the large-amplitude variability (Perna et al. 2006). Once the disk is gravitationally unstable, two classes of behavior are possible (e.g., Perna et al. 2006; Liu et al. 2014). First, the disk may develop a quasi-steady spiral structure which can transfer angular momentum outward and mass inward. Second, if the local cooling of the hyperaccretion disk is rapid, the disk may fragment into bound objects. In such case, a small fraction of the disk materials may break away from the main disk owing to the gravitational instability, and therefore can account for the central engine reactivity to power a bright X-ray flare.

After rapid accretion and/or merger of the initial fragments, the fragment mass can be estimated as (Takeuchi et al. 1996)

$$
M_{\mathrm{frag}} \simeq\left(\frac{H}{R}\right)^{2} \alpha^{1 / 2} M_{\mathrm{BH}},
$$

where $R$ is the distance from the accreting object, and $\alpha$ is a dimensionless parameter characterizing the strength of viscosity (Shakura \& Sunyaev 1973). Given $H \sim R$ and considering a range of the central compact object masses between 1.5 and $10 M_{\odot}$, Dall'Osso et al. (2017) showed fragments with masses on the order of $0.05 \sim 1 M_{\odot}$. The geometrical thickness of a GRB disk is between the standard thin disk and the advection-dominated accretion flow 
(e.g., Narayan \& Yi 1994). Thus, in this paper, $H / R$ can be roughly estimated in the range $0.1 \lesssim H / R \lesssim 0.2^{7}$ according to previous calculations on NDAF solutions (e.g., Popham et al. 1999; Kawanaka et al. 2013), particularly for the inner disk. In addition, the viscosity parameter is chosen as $\alpha=0.02$ according to MHD simulation results (Hirose et al. 2009).

The relationship between the fragment mass and BH mass is presented in Figure 6. According to previous simulations (Ruffert \& Janka 1997, 1999), the NS-NS merger may result in a BH around $2.5 \sim 3 M_{\odot}$, surrounded by a disk of around $0.1 \sim 0.2 M_{\odot}$. In Figure 6, we consider the $\mathrm{BH}$ masses in the range $2.5 \sim 20 M_{\odot}$. The solid lines represent the theoretical $M_{\text {frag }}$ for $H / R=0.1$ (blue) and 0.2 (red). As shown by the red line, the upper critical $M_{\text {frag }}$ at $M_{\mathrm{BH}}=3 M_{\odot}$ is $\sim 0.017 M_{\odot}$, which is a reasonable fraction (around 10\%) of the upper limit of the total disk mass $\left(0.1 \sim 0.2 M_{\odot}\right)$ during the NS-NS merger, according to previous simulations (e.g., Ruffert \& Janka 1997; Dietrich et al. 2015). For the BH-NS merger, the upper critical $M_{\text {frag }}$ is $\sim 0.057 M_{\odot}$, also around $10 \%$ of the upper limit of the total disk mass $\left(\sim 0.5 M_{\odot}\right.$ e.g., Kluźniak \& Lee 1998; Janka et al. 1999). On the other hand, it is known that the typical energy ratio of the X-ray flare to the prompt gamma-ray emission is around 10\% (e.g., Chincarini et al. 2010; Yi et al. 2016). Thus, the theoretical thresholds for $M_{\text {frag }}$ (the two solid lines in Figure 6) well agree with the observations and therefore may be reasonable.

For the two SGRBs, as shown in Figure 5, the fragment mass is around $0.02 M_{\odot}$ for the sum of two flares of GRB $131004 \mathrm{~A}$, and $0.025 M_{\odot}$ for the flare of GRB $161004 \mathrm{~A}$, as shown by the two dashed lines in Figure 6 . It is seen that these two values are just beyond the upper critical $M_{\text {frag }}$ under the NS-NS merger (at $M_{\mathrm{BH}} \sim 3 M_{\odot}$ ). On the contrary, the BH-NS merger can provide a larger fragment mass, i.e. up to $\sim 0.057 M_{\odot}$, so it can be responsible for the bright $\mathrm{X}$-ray flares. It is also seen from Figure 6 that, the two dashed lines (the parts between the two solid lines) indicate that the central BH masses of GRBs $131004 \mathrm{~A}$ and $161004 \mathrm{~A}$ are likely in the range $3.5 \sim 15 M_{\odot}$, corresponding to the BH-NS merger rather than the NS-NS merger.

However, we should stress that, due to the large uncertainty for the power and efficiency of the BZ process, the required fragment mass $M_{\text {frag }}$ for the X-ray flare is quite uncertain. The NS-NS merger may account for bright X-ray flares if the efficiency from the jet power to the jet luminosity is higher, or the $\mathrm{BH}$ spin is faster. In addition, even for the normal efficiency and spin parameter, the NS-NS merger can also be responsible for the flares with relatively low energy. Nevertheless, according to Equation (13), under the same parameters, the BH-NS merger will generally have a larger $M_{\text {frag }}$ than the NS-NS merger. We therefore argue that the BH-NS merger is more likely to be the central engine of GRBs with bright X-ray flares.

\section{CONCLUSIONS AND DISCUSSION}

In this work, we have studied a sample of 49 SGRBs with rapid Swift/XRT follow-up observations, and the adequate observational data in the early time. We have found that three bursts have four bright X-ray flares, among which three bright X-ray flares are probably related to the central engine reactivity. Since the GRB disk is thermally and viscously stable (Popham et al. 1999; Narayan et al. 2001; Di Matteo et al. 2002), the gravitational instability, possibly followed by fragmentation, may be the most likely candidate for the bright X-ray flares (Perna et al. 2006). Moreover, a comparison of the analytic BZ jet power with the observations is presented in this paper. We have found that, based on the BZ process, the fragment mass is sufficient for the required mass accretion for the bright flares in our sample. In our scenario, GRBs 131004A and 161004A are likely related to a massive disk around a BH, and therefore the BH-NS merger is more preferred than the NS-NS merger.

We should stress that our study is based on the assumption that the central engine is a BH hyper-accretion, formed by the BH-NS merger or the NS-NS merger. Apart from such a mechanism, a magnetar produced by the NS-NS merger can also be responsible for the central engine of the X-ray flares in SGRBs (e.g., Dai et al. 2006). It is well-known that the GW detectors advanced LIGO and Virgo have detected the GW radiation from the BH-BH and NS-NS mergers. We can expect that the GW radiation of BH-NS merger will also be detected in near future. Thus, our suggested link between the central engine-powered bright X-ray flare and the BH-NS merger can be checked.

The local event rate density for SGRBs varies slightly for different merger delay models: Gaussian, lognormal, and power-law delay models (Sun et al. 2015). It is known that the BH-NS merger rate is significantly lower than the NS-NS one. Voss \& Tauris (2003) shows that in our Galaxy the former is roughly $40 \%$ of the latter. In addition, in the present work we only consider the bright X-ray flares and ignore the possibility of dim flares originating from the BH-NS merger. Moreover, the disk mass during the BH-NS merger is sensitive to the mass ratio of the two compact

\footnotetext{
7 If the extreme case $H \sim R$ is used to estimate the fragment mass, and for a typical viscosity $\alpha=0.02$ and even a low-mass BH $M_{\mathrm{BH}}=3 M_{\odot}, M_{\text {frag }}$ is over $0.4 M_{\odot}$ according to Equation (13). Such a value is even beyond the total disk mass before fragment under the NS-NS merger, and therefore likely unphysical.
} 
objects. In other words, a BH-NS merger is likely a necessary but not a sufficient condition to have a massive disk. Thus, the percentage of the central engine-powered bright flares in our sample, $2 / 49 \simeq 4 \%$, is likely a reasonable value according to our suggested link.

We thank the referee for beneficial suggestions that improved the manuscript. We acknowledge the use of the public data from the Swift data archive, and the UK Swift Science Data Center. This work was supported by the National Basic Research Program of China (973 Program) under grants 2014CB845800, and the National Natural Science Foundation of China under grants 11573023, 11673062, 11473022, 11333004, 11503011, 11773007 and 11403005. W.M. Gu is supported by the CAS Open Research Program of Key Laboratory for the Structure and Evolution of Celestial Objects under grant OP201503. J. Mao is supported by the Hundred Talent Program, the Major Program of the Chinese Academy of Sciences (KJZD-EW-M06), and the Oversea Talent Program of Yunnan Province.

\section{REFERENCES}

Abbott, B. P., Abbott, R., Abbott, T. D., et al. 2016a, Physical Review Letters, 116, 061102

Abbott, B. P., Abbott, R., Abbott, T. D., et al. 2016b, Physical Review Letters, 116, 241103

Abbott, B. P., Abbott, R., Abbott, T. D., et al. 2017, Physical Review Letters, 119, 161101

Barnes, J., \& Kasen, D. 2013, ApJ, 775, 18

Barthelmy, S. D., Chincarini, G., Burrows, D. N., et al. 2005, Nature, 438, 994

Barthelmy, S. D., Evans, P. A., Gehrels, N., et al. 2016, GRB Coordinates Network, Circular Service, No. 20053, \#1 (2016), 20053, 1

Berger, E. 2014, ARA\&A, 52, 43

Bernardini, M. G., Margutti, R., Chincarini, G., Guidorzi, C., \& Mao, J. 2011, A\&A, 526, A27

Blandford, R. D., \& Znajek, R. L. 1977, MNRAS, 179, 433

Campana, S., Tagliaferri, G., Lazzati, D., et al. 2006, A\&A, 454, 113

Chincarini, G., Mao, J., Margutti, R., et al. 2010, MNRAS, 406, 2113

Dai, Z. G., \& Lu, T. 1998, A\&A, 333, L87

Dai, Z. G., Wang, X. Y., Wu, X. F., \& Zhang, B. 2006, Science, 311,1127

Dall'Osso, S., Perna, R., Tanaka, T. L., \& Margutti, R. 2017, MNRAS, 464, 4399

Di Matteo, T., Perna, R., \& Narayan, R. 2002, ApJ, 579, 706

Dietrich, T., Bernuzzi, S., Ujevic, M., \& Brügmann, B. 2015, PhRvD, 91, 124041

Eichler, D., Livio, M., Piran, T., \& Schramm, D. N. 1989, Nature, 340, 126

Evans, P. A., Beardmore, A. P., Page, K. L., et al. 2009, MNRAS, 397, 1177

Evans, P. A., Beardmore, A. P., Page, K. L., et al. 2007, A\&A, 469, 379

Giannios, D. 2006, A\&A, 455, L5

Goldstein, A., Veres, P., Burns, E., et al. 2017, ApJL, 848, L14

Grupe, D., Burrows, D. N., Patel, S. K., et al. 2006, ApJ, 653, 462

Hirose, S., Blaes, O., \& Krolik, J. H. 2009, ApJ, 704, 781

Ioka, K., Kobayashi, S., \& Zhang, B. 2005, ApJ, 631, 429

Janiuk, A., Mioduszewski, P., \& Moscibrodzka, M. 2013, ApJ, 776,105

Janiuk, A., Yuan, Y., Perna, R., \& Di Matteo, T. 2007, ApJ, 664,1011

Janka, H.-T., Eberl, T., Ruffert, M., \& Fryer, C. L. 1999, ApJL, 527, L39

Jin, Z.-P., Hotokezaka, K., Li, X., et al. 2016, Nature

Communications, 7, 12898

Jin, Z.-P., Li, X., Cano, Z., et al. 2015, ApJL, 811, L22
Just, O., Obergaulinger, M., Janka, H.-T., Bauswein, A., \& Schwarz, N. 2016, ApJL, 816, L30

Kasen, D., Metzger, B., Barnes, J., Quataert, E., \& Ramirez-Ruiz, E. 2017, Nature, 551, 80

Kawaguchi, K., Kyutoku, K., Nakano, H., et al. 2015, PhRvD, 92, 024014

Kawaguchi, K., Kyutoku, K., Shibata, M., \& Tanaka, M. 2016, ApJ, 825, 52

Kawanaka, N., Piran, T., \& Krolik, J. H. 2013, ApJ, 766, 31

Kluźniak, W., \& Lee, W. H. 1998, ApJL, 494, L53

Kobayashi, S., \& Zhang, B. 2007, ApJ, 655, 973

Kouveliotou, C., Meegan, C. A., Fishman, G. J., et al. 1993, ApJL, 413, L101

Kumar, P., \& Panaitescu, A. 2000, ApJL, 541, L51

Lee, H. K., Brown, G. E., \& Wijers, R. A. M. J. 2000a, ApJ, 536,416

Lee, H. K., Wijers, R. A. M. J., \& Brown, G. E. 2000b, PhR, 325,83

Lei, W.-H., Zhang, B., Wu, X.-F., \& Liang, E.-W. 2017, ApJ, 849,47

Li, L.-X., \& Paczyński, B. 1998, ApJL, 507, L59

Liang, E. W., Zhang, B., O'Brien, P. T., et al. 2006, ApJ, 646, 351

Liu, T., Gu, W.-M., \& Zhang, B. 2017, NewAR, 79, 1

Liu, T., Song, C.-Y., Zhang, B., Gu, W.-M., \& Heger, A. 2018, ApJ, 852, 20

Liu, T., Yu, X.-F., Gu, W.-M., \& Lu, J.-F. 2014, ApJ, 791, 69

Ma, S.-B., Lei, W.-H., Gao, H., et al. 2018, ApJL, 852, L5

Margutti, R., Chincarini, G., Granot, J., et al. 2011, MNRAS, 417,2144

Metzger, B. D. 2017, Living Reviews in Relativity, 20, 3

Metzger, B. D., \& Berger, E. 2012, ApJ, 746, 48

Metzger, B. D., Martínez-Pinedo, G., Darbha, S., et al. 2010, MNRAS, 406, 2650

Mu, H.-J., Gu, W.-M., Hou, S.-J., et al. 2016, ApJ, 832, 161

Narayan, R., Paczynski, B., \& Piran, T. 1992, ApJL, 395, L83

Narayan, R., Piran, T., \& Kumar, P. 2001, ApJ, 557, 949

Narayan, R., \& Yi, I. 1994, ApJL, 428, L13

Norris, J. P., Bonnell, J. T., Kazanas, D., et al. 2005, ApJ, 627 , 324

Paczynski, B. 1986, ApJL, 308, L43

Paczynski, B. 1991, AcA, 41, 257

Perna, R., Armitage, P. J., \& Zhang, B. 2006, ApJL, 636, L29

Popham, R., Woosley, S. E., \& Fryer, C. 1999, ApJ, 518, 356

Proga, D., \& Zhang, B. 2006, MNRAS, 370, L61

Rosswog, S. 2007, MNRAS, 376, L48

Ruffert, M., \& Janka, H.-T. 1997, Reviews in Modern Astronomy, 10, 201

Ruffert, M., \& Janka, H.-T. 1999, A\&A, 344, 573 
Savchenko, V., Ferrigno, C., Kuulkers, E., et al. 2017, ApJL, 848, L15

Shakura, N. I., \& Sunyaev, R. A. 1973, A\&A, 24, 337

Siegel, D. M., \& Metzger, B. D. 2017, Physical Review Letters, 119, 231102

Song, C.-Y., \& Liu, T. 2017, arXiv:1710.00142

Stamatikos, M., Barthelmy, S. D., Baumgartner, W. H., et al. 2013, GRB Coordinates Network, Circular Service, No. 15316, \#1 (2013), 15316, 1

Sun, H., Zhang, B., \& Li, Z. 2015, ApJ, 812, 33

Takeuchi, T., Miyama, S. M., \& Lin, D. N. C. 1996, ApJ, 460, 832

Tanaka, M., Hotokezaka, K., Kyutoku, K., et al. 2014, ApJ, 780, 31

Tauris, T. M., Kramer, M., Freire, P. C. C., et al. 2017, ApJ, 846,170
Voss, R., \& Tauris, T. M. 2003, MNRAS, 342, 1169

Woosley, S. E., \& Bloom, J. S. 2006, ARA\&A, 44, 507

Xue, L., Liu, T., Gu, W.-M., \& Lu, J.-F. 2013, ApJS, 207, 23

Yang, B., Jin, Z.-P., Li, X., et al. 2015, Nature Communications, 6, 7323

Yi, S.-X., Xi, S.-Q., Yu, H., et al. 2016, ApJS, 224, 20

Yu, Y.-W., Zhang, B., \& Gao, H. 2013, ApJL, 776, L40

Zhang, B. 2016, ApJL, 827, L31

Zhang, B., \& Mészáros, P. 2001, ApJL, 552, L35

Zhang, B., Zhang, B.-B., Virgili, F. J., et al. 2009, ApJ, 703, 1696

Zhang, B.-B., Zhang, B., Sun, H., et al. 2018, Nature Communications, 9,447 

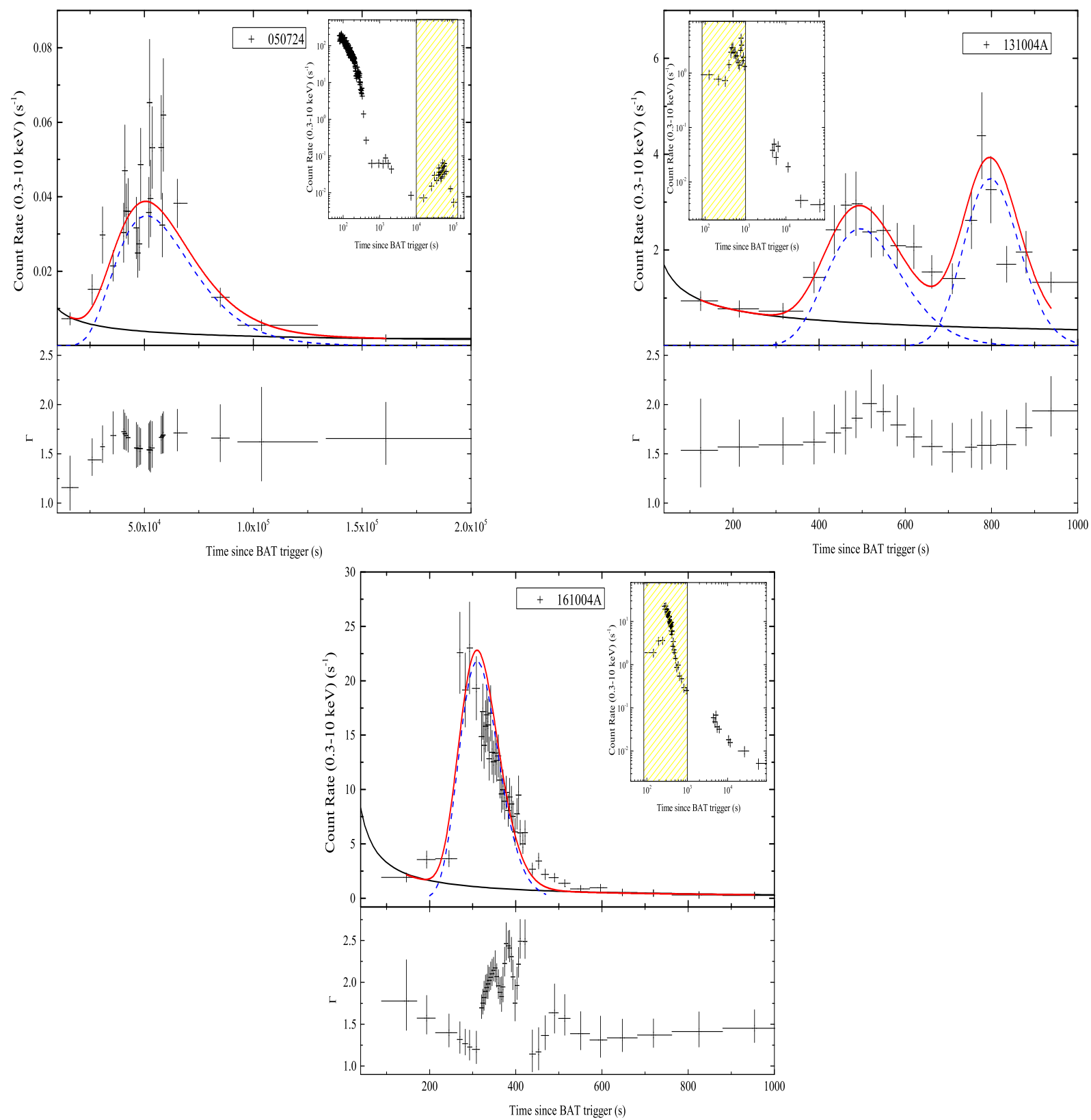

Figure 1. Upper panel: $0.3-10 \mathrm{keVcount}-$ rate light curve of the three SGRBs in our sample. Red solid line: best fitting for the total emission; blue dashed line: best fitting for the bright X-ray flare; black solid line: the smooth continuum contribution shown by a simple power-law. Insert: complete Swift/XRT light curve. The yellow area shows the total emission selected in our fitting. Lower panel: spectral photon index evolution. 


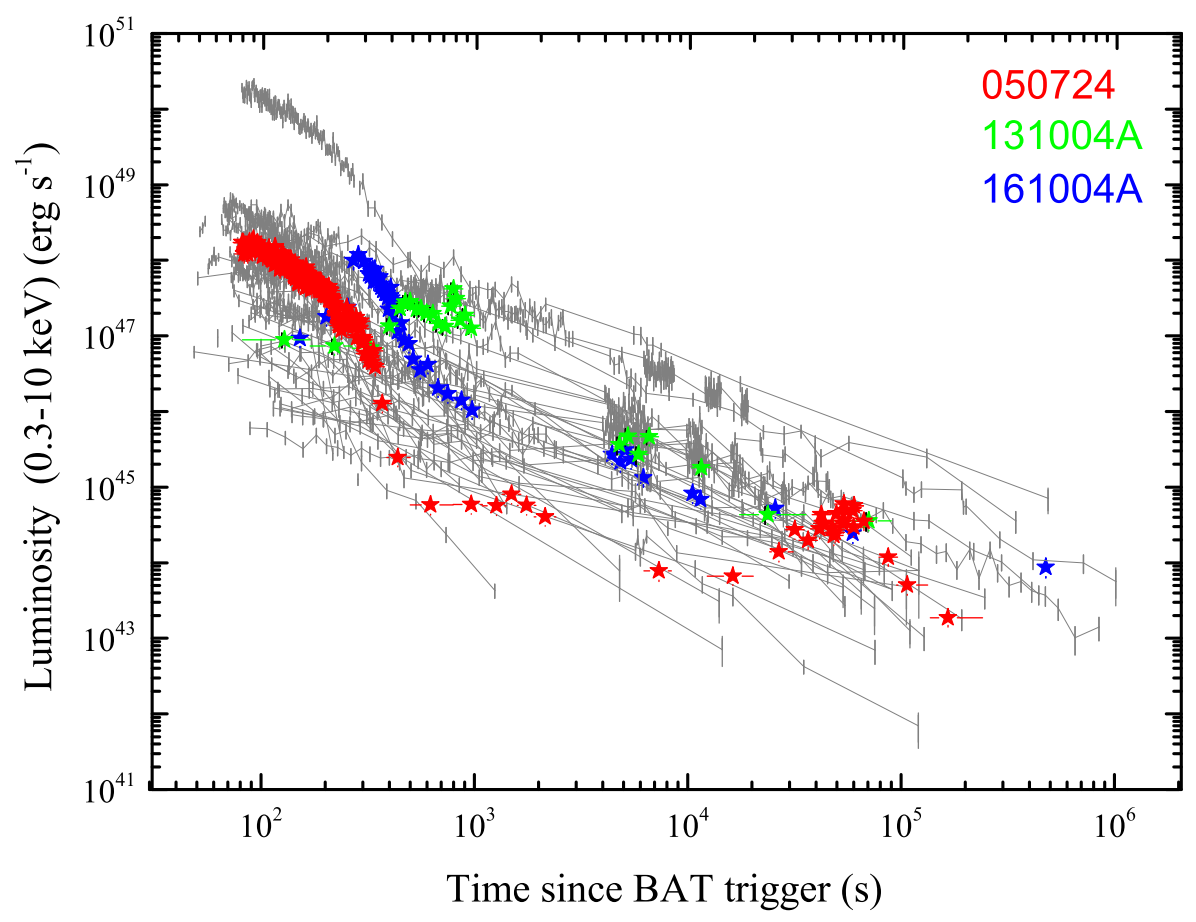

Figure 2. All the X-ray light curves of SGRBs in our sample. The three SGRBs $(050724,131004 \mathrm{~A}$ and 161004A) are shown by red, green and blue stars, respectively. The other sources are shown by gray curves. 


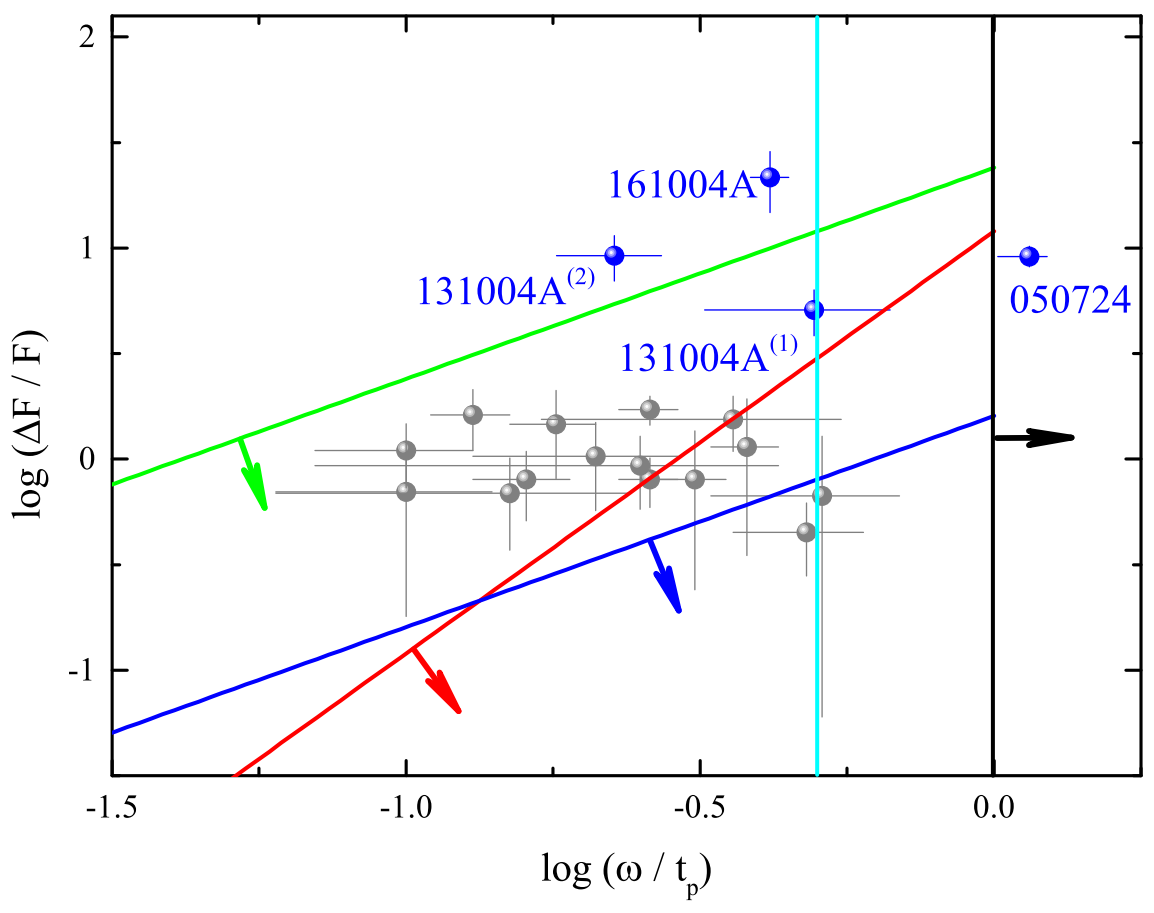

Figure 3. Relationship between the relative variability flux $\Delta F / F$ and the relative variability timescale $\omega / t_{\mathrm{p}}$ for the four bright X-ray flares in our SGRB sample. The bright flares in GRB 131004A and GRB 161004A are shown by the blue circles, compared to the dim X-ray flare candidates in short gamma-ray bursts (gray circles) from Margutti et al. (2011). The late-time bright X-ray flare detected in GRB $050724\left(t_{\mathrm{p}} \sim 5 \times 10^{4} \mathrm{~s}\right)$ is also shown by a blue circle. The four theoretical lines are identical with those in Figure 6 of Bernardini et al. (2011), i.e., density fluctuations on axis (blue line) and off-axis (red line), off-axis multiple regions density fluctuations (green line), patchy shell model (black line), see Ioka et al. (2005) for details. In addition, the condition $\omega / t_{\mathrm{p}}<0.5$ is shown by the left region to the cyan vertical line. 


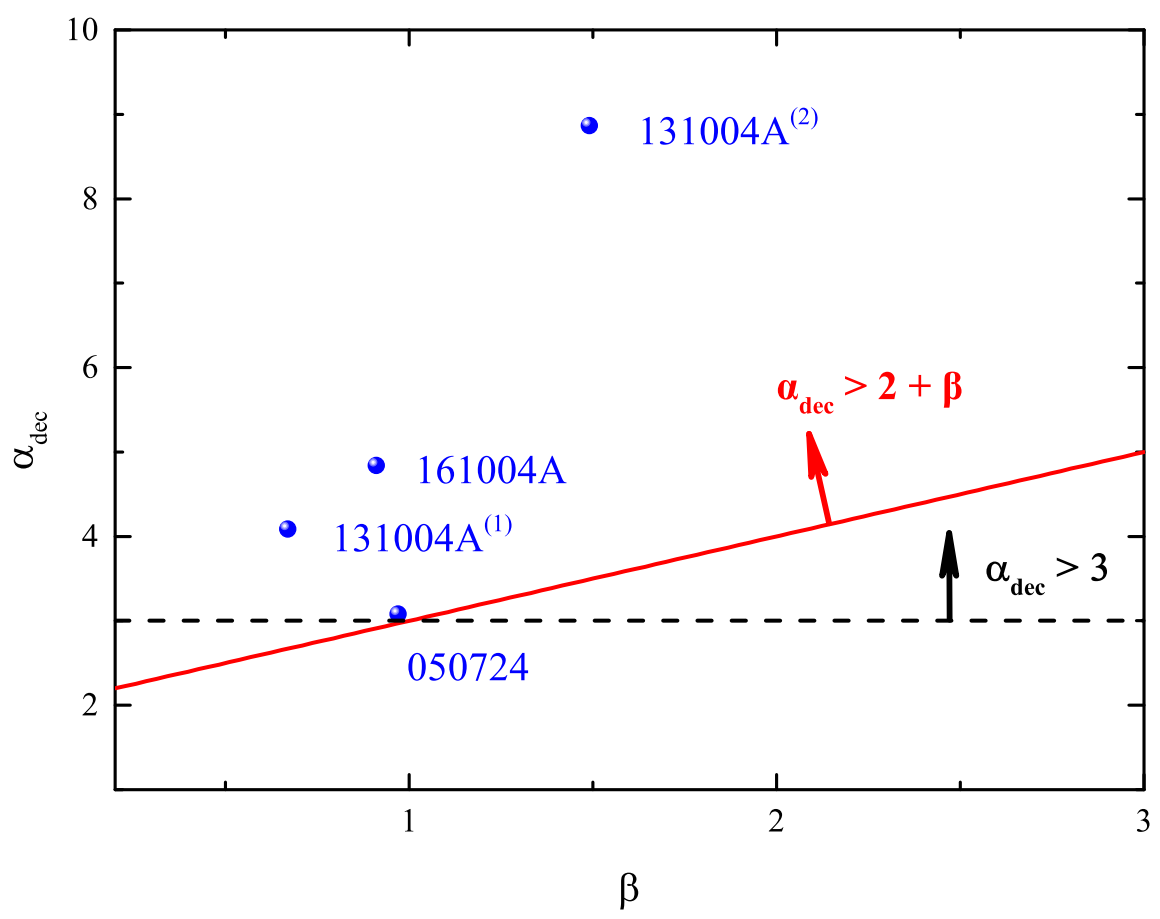

Figure 4. A comparison of the bright X-ray flares in our sample with the criterion of internal origin " $\alpha_{\text {dec }}>2+\beta$ " (the region above the red solid line) and " $\alpha_{\mathrm{dec}}>3$ " (the region above the black dashed line). 


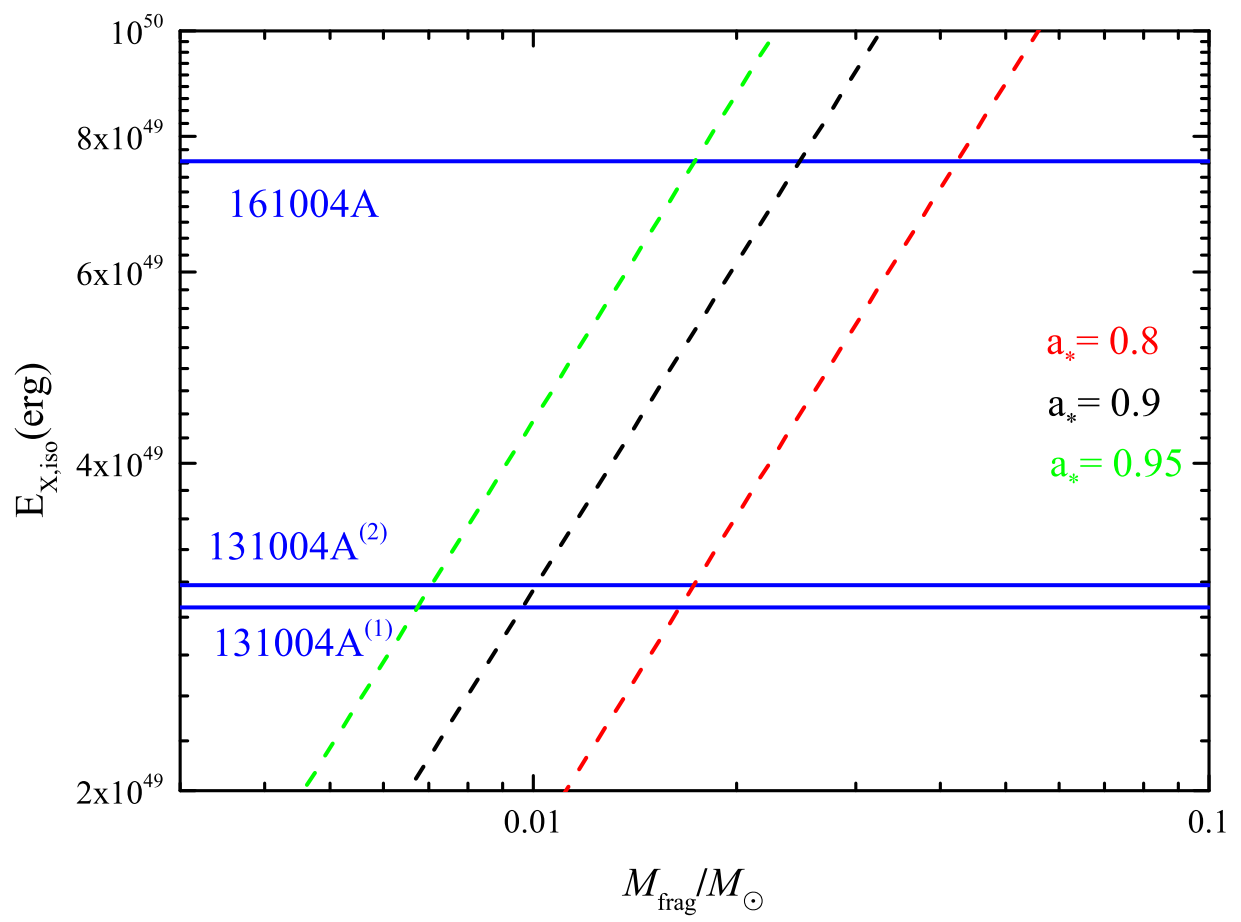

Figure 5. Relationship between the isotropic energy of the X-ray flares $E_{\mathrm{X} \text {,iso }}$ and the fragment mass $M_{\text {frag }}$ based on the BZ mechanism. The dashed lines represent the theoretical results according to Equation (9), where $a_{*}=0.8$ (red), 0.9 (black) and 0.95 (green). The isotropic energy $E_{\mathrm{X} \text {,iso }}$ for the flares from GRBs $131004 \mathrm{~A}$ and $161004 \mathrm{~A}$ are shown by the blue solid lines. 


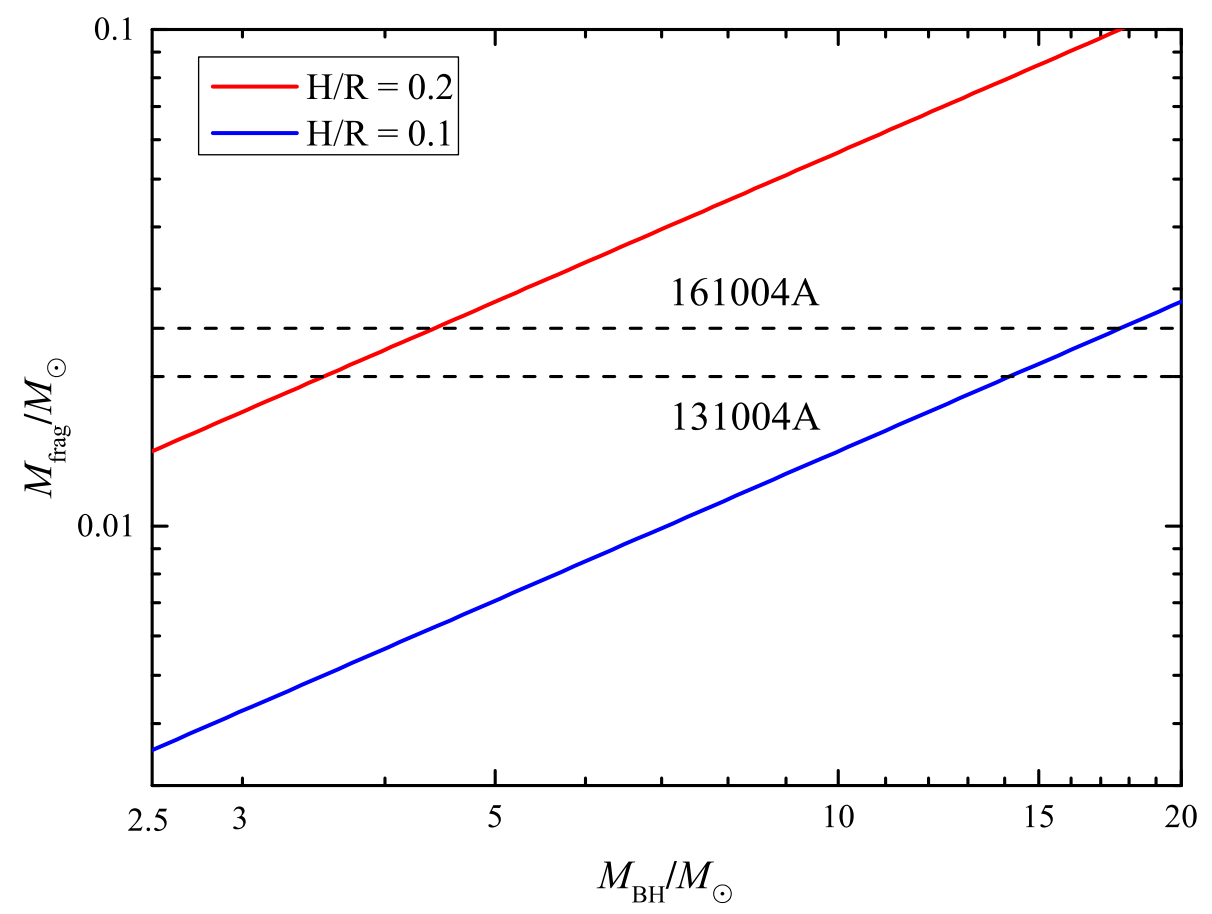

Figure 6. Relationship between the fragment mass $M_{\text {frag }}$ and the central $\mathrm{BH}$ mass $M_{\mathrm{BH}}$, where the viscosity parameter $\alpha=0.02$. The blue and red solid lines represent the theoretical results according to Equation $(13)$ for $H / R=0.1$ and 0.2 , respectively. The required fragment masses of the X-ray flares from GRBs 131004A and 161004A are shown by the black dashed lines, which are estimated by Equation (9) with a typical spin parameter $a_{*}=0.9$. 
Table 1. Fitting results of the flares in our sample.

\begin{tabular}{|c|c|c|c|c|c|c|c|c|c|c|c|}
\hline GRB & $\begin{array}{r}T_{90}{ }^{\mathrm{a}} \\
(\mathrm{s})\end{array}$ & $\begin{array}{c}A \\
\left(\text { counts s}^{-1}\right)\end{array}$ & $\begin{array}{c}\tau_{1} \\
\left(10^{4} \mathrm{~s}\right)\end{array}$ & $\begin{array}{l}\tau_{2} \\
(\mathrm{~s})\end{array}$ & $k$ & $\begin{array}{l}\omega \\
(\mathrm{s})\end{array}$ & $\omega / t_{\mathrm{p}}$ & $\Delta F / F$ & $\alpha_{\mathrm{dec}}$ & $\beta$ & $\begin{array}{c}N^{\mathrm{b}} \\
\left(\operatorname{erg~} \mathrm{cm}^{-2}\right)\end{array}$ \\
\hline $050724^{\mathrm{c}}$ & 3 & $0.035 \pm 0.003$ & $21.6 \pm 4.62$ & $(1.2 \pm 0.2) \times 10^{4}$ & 0.12 & $5.09 \times 10^{4}$ & $1.15 \pm 0.19$ & $9.12 \pm 3.40$ & 3.08 & 0.97 & - \\
\hline $131004 \mathrm{~A}^{(1) \mathrm{d}, \mathrm{f}}$ & 1.54 & $2.45 \pm 0.53$ & $0.82 \pm 0.41$ & $29.77 \pm 14.71$ & 0.06 & 244.7 & $0.49 \pm 0.17$ & $5.08 \pm 1.24$ & 4.09 & 0.67 & $4.02 \mathrm{E}-11$ \\
\hline $131004 \mathrm{~A}^{(2) \mathrm{e}}$ & 1.54 & $3.50 \pm 0.83$ & $6.26 \pm 1.82$ & $10.16 \pm 2.87$ & 0.10 & 180.2 & $0.23 \pm 0.05$ & $9.22 \pm 2.24$ & 8.86 & 1.49 & $4.02 \mathrm{E}-11$ \\
\hline $161004 \mathrm{~A}^{\mathrm{g}}$ & $1.3-3$ & $21.8 \pm 3.7$ & $0.72 \pm 0.08$ & $13.29 \pm 1.33$ & 0.24 & 129.1 & $0.42 \pm 0.03$ & $21.67 \pm 6.93$ & 4.84 & 0.91 & $4.64 \mathrm{E}-11$ \\
\hline
\end{tabular}

$a_{\text {duration in the } 15-350 \mathrm{keV} \text { band. }}$

${ }^{b} N$ is the factor of count-rate light curves converting into flux, taking GRB $161004 \mathrm{~A}$ as an example, $1 \mathrm{count}=4.64 \times 10^{-11} \mathrm{erg} \mathrm{cm}^{-2}$.

${ }^{c}$ See Barthelmy et al. (2005); Campana et al. (2006); Grupe et al. (2006) for GRB 050724.

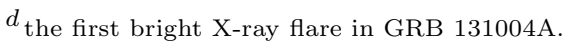

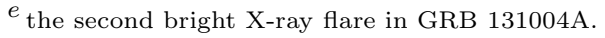

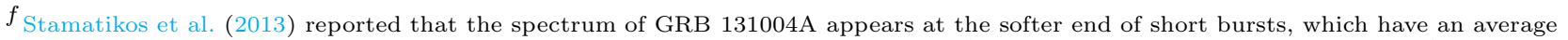
power-law spectral index of 1.2 . The spectral lag for the burst is $0.130 \mathrm{~s} \pm 0.020 \mathrm{~s}$ (for the $50-100 \mathrm{keV}$ and $15-25 \mathrm{keV}$ bands), which is significantly longer than the regular SGRBs.

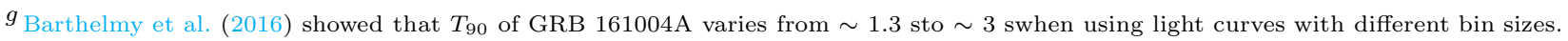


Table 2. Physical parameters of the flares.

\begin{tabular}{lcccc}
\hline \hline \multicolumn{1}{c}{ GRB } & $z$ & $\begin{array}{c}\omega_{\text {rest }}{ }^{\mathrm{a}} \\
(\mathrm{s})\end{array}$ & $\begin{array}{c}E_{\mathrm{X}, \text { iso }}{ }^{\mathrm{b}} \\
\left(10^{49} \mathrm{erg}\right)\end{array}$ & $\begin{array}{c}L_{\mathrm{p}}{ }^{\mathrm{c}} \\
\left(10^{47} \mathrm{erg} \mathrm{s}^{-1}\right)\end{array}$ \\
\hline 050724 & 0.257 & $4.049 \times 10^{4}$ & - & - \\
\hline $131004 \mathrm{~A}^{(1)}$ & 0.717 & 142.5 & 2.95 & $2.32 \pm 0.51$ \\
$131004 \mathrm{~A}^{(2)}$ & 0.717 & 104.9 & 3.09 & $3.32 \pm 0.79$ \\
$161004 \mathrm{~A}$ & - & 86.09 & 7.59 & $9.92 \pm 1.67$ \\
\hline
\end{tabular}

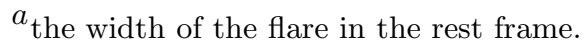

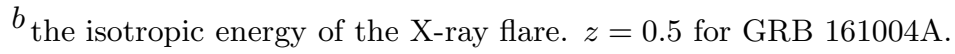

${ }^{c}$ the isotropic peak luminosity of the flare. 